\title{
FDG PET/CT metabolic tumour volume in small-cell lung cancer: better staging and prognostic stratification for an improved therapeutic strategy
}

\author{
Giovanni Luca Ceresoli
}

Published online: 17 March 2012

(C) Springer-Verlag 2012

Small-cell lung cancer (SCLC) represents $15-20 \%$ of all diagnosed cases of lung cancer. It is characterized by distinct clinical features, such as rapid tumour growth, predominantly central and bulky location in the chest, and early dissemination to locoregional lymph nodes and to distant metastatic sites [1]. Despite the high initial response rates to chemotherapy and the high sensitivity to radiotherapy (RT), SCLC recurs in most patients, and prognosis remains generally poor. About two-thirds of patients present with overt metastatic disease, and for these patients combined chemotherapy is the cornerstone of treatment. Unfortunately, no curative option is available, and median survival is less than 1 year $[2,3]$. For the minority of patients with limited intrathoracic disease at diagnosis, chemotherapy combined with thoracic RT is the standard approach, with a definite chance of cure in about $20-25 \%$ of patients [4]. In both limited and metastatic disease with a major response to chemotherapy, prophylactic cranial irradiation has been shown to reduce the rate of brain recurrence and to prolong survival $[5,6]$.

Although chemotherapy remains the backbone of treatment in SCLC, beneficial effects on survival in the past two decades have been mainly achieved by the appropriate use of RT [7]. A number of randomized studies have demonstrated a survival advantage when thoracic RT is administered early during the chemotherapy course [8], and in particular, a shorter interval between the first day of chemotherapy and the last day of RT is associated with improved survival due to the reduced risk of tumour repopulation and

\section{G. L. Ceresoli $(\bowtie)$}

Thoracic and GU Oncology Unit, Humanitas Gavazzeni Clinic,

Via M. Gavazzeni, 21,

24125 Bergamo, Italy

e-mail: giovanni_luca.ceresoli@gavazzeni.it development of resistant clones [9]. Twice-daily thoracic RT starting with the first course of chemotherapy results in improved survival rates in comparison to standard oncedaily RT [10], and preliminary studies have shown that higher doses exceeding 45-54 Gy can be safely delivered concurrently with chemotherapy [11]. However, early RT, twice-daily fractionation and higher doses are all related to higher toxicity, mainly severe oesophagitis. A reasonable approach to reducing toxicity and to improving locoregional control and survival is, on the one hand, accurate staging and patient selection, and on the other hand, better delineation of RT target volumes.

Patients with SCLC have been traditionally staged by a two-stage system, developed nearly 50 years ago by the Veterans Administration Lung Cancer Study Group [12]. According to this staging system, limited disease (LD) is defined as disease confined to the ipsilateral hemithorax which can be encompassed within a tolerable radiation field, whereas extensive disease (ED) is defined as a disease extending outside the ipsilateral hemithorax, including patients with malignant pleural effusion. More recently, the International Association for the Study of Lung Cancer has recommended that TNM staging be applied in all cases of SCLC, and that accurate stratification by TNM stage be incorporated into clinical trials in stage I/III $[13,14]$.

A precise definition of target volumes is crucial in RT to avoid geographic misses and to limit exposure of healthy tissues to high doses of radiation. Mediastinal staging is of paramount importance, mainly if increased RT doses are considered. Studies in non-small-cell lung cancer (NSCLC) have clearly demonstrated that dose escalation is possible if elective nodal irradiation of the whole mediastinal area is omitted. CT-based selective irradiation of involved lymph nodes only has been proven safe in NSCLC, with isolated nodal failures in less than $5 \%$ of patients [15]. This has 
resulted in reduced radiation fields. Unfortunately, this does not seem to be the case in SCLC. Isolated nodal recurrence has been seen in over $10 \%$ of patients in whom only lymph nodes seen to be enlarged on CT were irradiated [16]. This is in agreement with the poor correlation between clinical and pathological staging observed in the largest reported series of resected SCLC [14]. In that series, $14 \%$ of 144 patients with clinical stage N0 according to CT were upstaged to pN2 or above after resection; conversely, $32 \%$ of those with clinical N2 stage were downstaged to $\mathrm{pN} 1$ or less.

${ }^{18} \mathrm{~F}$-FDG PET has been demonstrated to be of significant value in NSCLC, improving the definition of the primary tumour, mainly in regions with atelectasis, and of nodal disease, leading to reduced normal tissue toxicity and providing opportunities for dose escalation [17]. Therefore, an FDG PET/CT scan is now recommended as the optimal procedure for patient selection and RT planning in patients with locally advanced NSCLC [18].

Preliminary data on the use of FDG PET in SCLC are encouraging, albeit still not conclusive. In several small studies evaluating staging of SCLC, FDG PET has shown higher accuracy than conventional imaging (including bone scintigraphy and brain imaging), with stage migration in a median of $13 \%$ of patients, upstaging from LD to ED in $9 \%$ and downstaging from ED to $\mathrm{LD}$ in $4 \%$ [19]. However, because SCLC is rarely managed with surgery, all these studies have the important limitation that no histological verification of discordant results between conventional imaging and FDG PET was available. FDG PET is also being rapidly integrated into RT planning procedures for LD SCLC. In one study, FDG PET changed RT fields in $24 \%$ of patients with LD; both increases and decreases in the volumes were observed [20]. On the basis of this planning study, 60 patients with LD were prospectively treated with combined chemotherapy/RT, with irradiation of the primary tumour and nodal mediastinal disease defined by pretreatment FDG PET/CT imaging [21]. Isolated nodal failures were observed in only $3 \%$ of patients, and the incidence of severe oesophagitis was $12 \%$, that is half of that expected with the use of CT only.

While its additional value in the assessment of early response to therapy in SCLC is questionable [22], due to the rapidly visible response on CT, FDG PET has recently shown promising results as a pretreatment tool for patient prognostic stratification. In a subgroup analysis of 76 consecutive patients with SCLC, overall survival (OS) was significantly shorter in those with high versus low mean SUVmax, including both those with LD and those with ED [23]. More recently, following a retrospective evaluation of a small series of SCLC patients, it was suggested that volumetric parameters such as total lesion glycolysis could be a better prognostic indicator than SUVmax, as reported for other cancers [24]. Zhu et al. [25] confirmed this finding in a larger retrospective series of patients with pathologically proven SCLC who underwent pretreatment FDG PET/ CT. In their study, the prognostic significance of several metabolic parameters was evaluated. On multivariate analysis, metabolic tumour volume (MTV) measured according to a threshold SUV value of 2.5 and integrated SUV (iSUV), defined as the product of MTV and average SUV, were significantly correlated with OS and progression-free survival (PFS), while no correlation with patient outcome was observed for SUVmax. Patients with LD and larger MTV showed significantly shorter OS and PFS as compared with those with LD and smaller MTV. Patients with ED and larger MTV also had significantly shorter median OS and PFS than patients with the same stage and smaller MTV.

In this issue of the European Journal of Nuclear Medicine and Molecular Imaging, Oh et al. [26] provide another useful piece of information about the potential of volumetric FDG PET evaluation in staging and prognostic definition of SCLC, and ultimately in the selection of appropriate therapy for each patient group. Their uni- and multivariate analyses of a retrospective series of 106 consecutive SCLC patients (45 LD, $61 \mathrm{ED}$ ) showed that both stage and whole-body MTV (measured according to a threshold SUV of 3) were independent prognostic factors for PFS and OS. Moreover, incorporation of PET-defined MTV with TNM staging provided a more detailed prediction of prognosis than MTV with conventional staging as well as tumour staging alone. These findings are very interesting, and suggest that FDG PET/CT may have an important role in patient stratification in future clinical trials and, hopefully, in clinical practice in SCLC. As discussed by the authors, a more accurate survival prognostication including whole-body MTV could help to improve treatment strategy for SCLC patients. In particular, patients with stage I/III SCLC and a lower whole-body MTV could be good candidates for RT dose escalation or accelerated and hyperfractionated regimens, while patients with stage IV SCLC but a lower disease burden could be candidates for consolidation thoracic and extrathoracic RT. These are exactly the issues explored by currently ongoing key RT trials in SCLC, which have been designed on CT-based staging and target definition [7]. Two randomized phase III studies are comparing high-dose, once-daily RT (66-70 Gy over 7 weeks) with 45 Gy in 30 twice-daily fractions over 3 weeks [27, 28]. On the other hand, two randomized trials have recently been launched in ED SCLC, to determine the role of consolidation RT in thoracic disease or limited metastatic areas in patients who are good responders to induction chemotherapy [29, 30]. Of note, a significant improvement in survival with the use of thoracic RT in patients with ED SCLC and a complete response at distant sites following chemotherapy was observed in a previous randomized study [31]. 
In conclusion, although a number of technical and biological issues (such as SUV threshold and organ motion) are still under debate and await standardization, the results reported by $\mathrm{Oh}$ et al. represent an important step towards the full implementation of FDG PET in SCLC. Further controlled prospective studies in a larger number of patients are warranted to confirm the role of metabolic volumetric parameters as staging tools and prognostic biomarkers in SCLC.

\section{References}

1. van Meerbeeck JP, Fennell DA, De Ruysscher DK. Small-cell lung cancer. Lancet. 2011;378:1741-55.

2. Mascaux C, Paesmans M, Berghmans T, Branle F, Lafitte JJ, Lemaitre $\mathrm{F}$, et al. A systematic review of the role of etoposide and cisplatin in the chemotherapy of small cell lung cancer with methodology assessment and meta-analysis. Lung Cancer. 2000;30:23-36

3. Abidin AZ, Garassino MC, Califano R, Harle A, Blackhall F. Targeted therapies in small cell lung cancer: a review. Ther Adv Med Oncol. 2010;2:25-37.

4. Lally BE, Urbanic JJ, Blackstock AW, Miller AA, Perry MC. Small cell lung cancer: have we made any progress over the last 25 years? Oncologist. 2007;12:1096-104.

5. Le Péchoux C, Dunant A, Senan S, Wolfson A, Quoix E, FaivreFinn $\mathrm{C}$, et al. Standard-dose versus higher-dose prophylactic cranial irradiation (PCI) in patients with limited-stage small-cell lung cancer in complete remission after chemotherapy and thoracic radiotherapy (PCI 99-01, EORTC 22003-08004, RTOG 0212, and IFCT 99-01): a randomised clinical trial. Lancet Oncol. 2009; 10:467-74.

6. Slotman B, Faivre-Finn C, Kramer G, Rankin E, Snee M, Hatton $\mathrm{M}$, et al. Prophylactic cranial irradiation in extensive small-cell lung cancer. N Engl J Med. 2007;357:664-72.

7. Slotman BJ, Senan S. Radiotherapy in small-cell lung cancer: lessons learned and future directions. Int J Radiat Oncol Biol Phys. 2011;79:998-1003.

8. Fried DB, Morris DE, Poole C, Rosenman JG, Halle JS, Detterbeck $\mathrm{FC}$, et al. Systematic review evaluating the timing of thoracic radiation therapy in combined modality therapy for limited-stage smallcell lung cancer. J Clin Oncol. 2004;22:4837-45.

9. De Ruysscher D, Pijls-Johannesma M, Bentzen SM, Minken A, Wanders R, Lutgens L, et al. Time between the first day of chemotherapy and the last day of chest radiation is the most important predictor of survival in limited-disease small-cell lung cancer. J Clin Oncol. 2006;24:1057-63.

10. Turrisi 3rd AT, Kim K, Blum R, Sause WT, Livingston RB, Komaki R, et al. Twice-daily compared with once-daily thoracic radiotherapy in limited small-cell lung cancer treated concurrently with cisplatin and etoposide. N Engl J Med. 1999;340:265-71.

11. Komaki R, Swann S, Ettinger D, Glisson BS, Sandler AB, Movsas $\mathrm{B}$, et al. Phase I study of thoracic radiation dose escalation with concurrent chemotherapy for patients with limited small cell lung cancer: report of Radiation Therapy Oncology Group (RTOG) protocol 97-12. Int J Radiat Oncol Biol Phys. 2005;62:342-50.

12. Micke P, Faldum A, Metz T, Beeh KM, Bittinger F, Hengstler JG, et al. Staging small cell lung cancer: Veterans Administration Lung Study Group versus International Association for the Study of Lung Cancer. What limits limited disease. Cancer. 2003;37:271-6.
13. Shepherd FA, Crowley J, van Houtte P, Postmus PE, Carney D, Chansky K, et al. The International Association for the Study of Lung Cancer Lung Cancer Staging Project: proposal regarding the clinical staging of small cell lung cancer in the forthcoming (seventh) edition of the Tumor, Node, Metastasis classification for lung cancer. J Thorac Oncol. 2007;2:1067-77.

14. Vallières E, Shepherd FA, Crowley J, Van Houtte P, Postmus PE, Carney D, et al. The IASLC Lung Cancer Staging Project. Proposals regarding the relevance of TNM in the pathologic staging of small cell lung cancer in the forthcoming (seventh) edition of the TNM classification for lung cancer. J Thorac Oncol. 2009;4:104959.

15. De Ruysscher D, Faivre-Finn C, Nestle U, Hurkmans CW, Le Péchoux C, Price A, et al. European Organization for Research and Treatment of Cancer (EORTC) recommendations for planning and delivery of high-dose, high-precision radiotherapy for lung cancer. J Clin Oncol. 2010;28:5301-10.

16. De Ruysscher D, Bremer RH, Koppe F, Wanders S, van Haren E, Hochstenbag $\mathrm{M}$, et al. Omission of elective nodal irradiation on basis of CT-scans in patients with limited disease small cell lung cancer: a phase II trial. Radiother Oncol. 2006;80:307-12.

17. van Loon J, van Baardwijk A, Boersma L, Ollers M, Lambin P, De Ruysscher D. Therapeutic implications of molecular imaging with PET in the combined modality treatment of lung cancer. Cancer Treat Rev. 2011;37:331-43.

18. De Ruysscher D, Nestle U, Jeraj R, MacManus M. PET scans in radiotherapy planning of lung cancer. Lung Cancer. 2012;75:1415.

19. Thomson D, Hulse P, Lorigan P, Faivre-Finn C. The role of positron emission tomography in management of small cell lung cancer. Lung Cancer. 2011;73:121-6.

20. van Loon J, Offermann C, Bosmans G, Wanders R, Dekker A, Borger $\mathrm{J}$, et al. 18FDG-PET radiation planning of mediastinal lymph nodes in limited disease small cell lung cancer changes radiotherapy fields: a planning study. Radiother Oncol. 2008;87:49-54

21. van Loon J, De Ruysscher D, Wanders R, Boersma L, Simons J, Oellers M, et al. Selective nodal irradiation on basis of 18FDGPET scans in limited disease small cell lung cancer: a phase II trial. Int J Radiat Oncol Biol Phys. 2010;77:329-36.

22. Fischer BM, Mortensen J, Langer SW, Loft A, Berthelsen AK, Daugaard $G$, et al. PET/CT imaging in response evaluation of patients with small cell lung cancer. Lung Cancer. 2006;54:41-9.

23. Lee YJ, Cho A, Cho BC, Yun M, Kim SK, Chang J, et al. High tumor metabolic activity as measured by fluorodeoxyglucose positron emission tomography is associated with poor prognosis in limited and extensive stage small-cell lung cancer. Clin Cancer Res. 2009;15:2426-32.

24. Arslan N, Tuncel M, Kuzhan O, Alagoz E, Budakoglu B, Ozet A, et al. Evaluation of outcome prediction and disease extension by quantitative 2-deoxy-2-18F-fluoro-D-glucose with positron emission tomography in patients with small cell lung cancer. Ann Nucl Med. 2011;25:406-13.

25. Zhu D, Ma T, Niu Z, Zheng J, Han A, Zhao S, et al. Prognostic significance of metabolic parameters measured by $18 \mathrm{~F}$ fluorodeoxyglucose positron emission tomography/computed tomography in patients with small cell lung cancer. Lung Cancer. 2011;73:332-7.

26. Oh JR, Seo JH, Chong A, Min JJ, Song HC, Kim YC, et al. Whole-body metabolic tumour volume of 18F-FDG PET/CT improves the prediction of prognosis in small cell lung cancer. Eur J Nucl Med Mol Imaging. 2012. doi:10.1007/s00259-0112059-7.

27. National Institutes of Health. A 2-arm randomized controlled trial of concurrent chemo-radiotherapy comparing twice-daily and once-daily radiotherapy schedules in patients with limited stage 
small cell lung cancer (SCLC) and good performance status [CONVERT]. NCT00433563. http://www.clinicaltrials.gov/ct2/show/ NCT00433563?term $=$ NCT00433563\&rank $=1$.

28. National Institutes of Health. Phase III comparison of thoracic radiotherapy regimens in patients with limited small cell lung cancer also receiving cisplatin and etoposide. NCT00632853. http://www.clinical trials.gov/ct2/show/NCT00632853?term=NCT00632853\&rank=1.

29. Nederlands Trial Register. Randomised trial on chest irradiation in extensive disease small cell lung cancer. NTR1527. http://www. trialregister.nl/trialreg/admin/rctview.asp? $\mathrm{TC}=1527$.
30. National Institutes of Health. Randomized phase II study comparing prophylactic cranial irradiation alone to prophylactic cranial irradiation and consolidative extra-cranial irradiation for extensive disease small cell lung cancer (ED-SCLC). NCT01055197. http:// www.clinicaltrials.gov/ct2/show/NCT01055197?term $=$ Phase + II+cranial+irradiation+ prophylactic+small+cell+lung+cancer.

31. Jeremic B, Shibamoto Y, Nikolic N, Milicic B, Milisavljevic S, Dagovic A, et al. Role of radiation therapy in the combinedmodality treatment of patients with extensive disease small-cell lung cancer: a randomized study. J Clin Oncol. 1999;17:2092-9. 